\title{
Skin resurfacing procedures: new and emerging options
}

This article was published in the following Dove Press journal:

Clinical, Cosmetic and Investigational Dermatology

28 August 2014

Number of times this article has been viewed

\author{
Mathew M Loesch' \\ Ally-Khan Somani' \\ Melanie M Kingsley' \\ Jeffrey B Travers ${ }^{1-3}$ \\ Dan F Spandau ${ }^{1,4}$ \\ 'Department of Dermatology, \\ 2Department of Pharmacology and \\ Toxicology, ${ }^{3}$ Department of Pediatrics, \\ ${ }^{4}$ Department of Biochemistry and \\ Molecular Biology, Indiana University \\ School of Medicine, Indianapolis, IN, \\ USA
}

Correspondence: Dan F Spandau Indiana University School of Medicine, 975 West Walnut Street, Room 349, Indianapolis, IN 46202, USA

Email dspanda@iu.edu

\begin{abstract}
The demand for skin resurfacing and rejuvenating procedures has progressively increased in the last decade and has sparked several advances within the skin resurfacing field that promote faster healing while minimizing downtime and side effects for patients. Several technological and procedural skin resurfacing developments are being integrated into clinical practices today allowing clinicians to treat a broader range of patients' skin types and pathologies than in years past, with noteworthy outcomes. This article will discuss some emerging and developing resurfacing therapies and treatments that are present today and soon to be available.
\end{abstract}

Keywords: rejuvenation, wounding, non-melanoma skin cancer, therapy

\section{Introduction}

Medical and esthetic skin procedures have seen a steady surge within the last decade largely stemming from greater numbers of new skin cancer diagnoses and a higher demand for skin rejuvenation practices. In 2013 in the United States, dermatologic surgeons performed over 9.5 million treatments, an almost $22 \%$ increase from the previous year, with a rising number of treatments involving skin resurfacing in the areas of laser/light/energy-based procedures (2.25 million), chemical peels ( 1.1 million), and microdermabrasion $(974,000) .{ }^{1}$ With this greater demand for skin resurfacing, selecting an appropriate treatment modality for the patient's skin type and condition is critical for a successful skin resurfacing therapy. Exploration to uncover the next generation of skin resurfacing treatments is being pursued to engineer therapies that further improve patients' skin texture and appearance with minimal downtime and complications. The purpose of this review is to highlight some of the research that has been conducted into emerging resurfacing therapies being explored and delivered in practice today.

\section{Ablative laser resurfacing background}

Treatment of skin disorders using laser technologies dates back to the early 1960s, and quickly became a popular method for improving facial wrinkles, scars, skin growths, and blemishes. ${ }^{2}$ Laser-based tissue destruction is based on the characteristics of laser wavelength, chromophore targeting, energy density, and laser pulse duration such that when these parameters are optimized, appropriate energy is delivered for targeted laser resurfacing with minimal collateral tissue damage. ${ }^{3}$ In the $1980 \mathrm{~s}$, continuous wave $\mathrm{CO}_{2}$ lasers became available for more effective esthetic resurfacing, but resulted in mixed 
outcomes that included many anticipated side effects following resurfacing treatment. ${ }^{4}$ Early $\mathrm{CO}_{2}$ resurfacing lasers generated a high rate of dyspigmentation, scarring, erythema, skin eruptions and infections as well as increased pain, discomfort, and downtime. The enhanced thermal injury was in part due to the continuous wave nature of these early $\mathrm{CO}_{2}$ lasers that left a wide zone of thermal damage and ablation. ${ }^{5}$ Later, pulsed and scanned $\mathrm{CO}_{2}$ laser systems, along with erbium-doped yttrium aluminum garnet (Er:YAG) lasers were developed to lessen the thermal damage to treated skin tissue. The reduction in thermal injury was a result of better precision with ablation to the intended target depths, and imparting minimal thermal injury to uninvolved skin. Currently, the Er:yttrium scandium gallium garnet laser (YSGG) by Cutera (Pearl, Cutera, Inc., Brisbane, CA, USA) has furthered this ablative laser evolution with technology that better enables a balance of depth and thermal impact not achievable with either of the other ablative wavelengths. ${ }^{6}$ Yet, the $\mathrm{CO}_{2}$ laser has remained the gold standard for skin resurfacing and rejuvenation. ${ }^{7}$

In the early 2000 s, further laser development made available the ablative fractional laser (AFL) that enabled delivery of skin treatments with a grid of vertical columns known as micro-thermal treatment zones (MTZ), which penetrated the epidermis and dermis. ${ }^{8}$ These MTZs have a diameter and depth that are dependent upon the settings of the fractional laser being used. What makes this method unique is that the undamaged skin adjacent to the MTZ provides a reservoir of viable tissue that allows for repopulation of the thermally destroyed and missing epidermis and dermis. ${ }^{9}$ Also, the dermal penetration by the fractional lasers leads to new and remodeled collagen formation by the surrounding fibroblasts, as well as the tightening of existing collagen that contributes to the filling of wrinkles, reduced facial rhytides, and improved skin tone. Overall, the fractional laser treatment results in faster healing, shorter downtime, and less adverse effects in comparison to continuous ablative laser modalities. Further developments in the last decade have made several different technologies available in the field of laser skin resurfacing. ${ }^{10}$ Many of these advances have been in the area of combination therapies such as incorporating either continuous wave or fractional resurfacing systems with other treatments. Some of these emerging therapies which will be reviewed here include laser-radiofrequency therapy, platelet rich plasma incorporation with laser ablation, and laser assisted drug delivery.

\section{Lasers and topical drug application}

Topical drug application has significant advantages over systemic therapy in treating skin diseases due to the ability to directly target the affected skin. ${ }^{11}$ Topical application also reduces possible side effects, toxicities, and drug degradation that are commonly seen with hepatic metabolism and systemic drug circulation. Despite the advantages of topical medication, the skin's barrier function still limits the application of most medications. ${ }^{12}$ With the kinetics of topical drug delivery dependent upon the laws of diffusion, drugs penetrate into the skin through two routes: the transepidermal penetration through intercellular and intracellular pathways, and the transappendageal penetration of sweat glands and hair follicles. Using either route, it is at the stratum corneum layer where resistance to drug diffusion is at its highest and has garnered interest by researchers to discover new methods of increasing cutaneous drug penetration. ${ }^{13}$ One new method being studied to enhance drug delivery is using the combination of topical medications and AFL resurfacing. The photothermolysis by AFLs allows vertical column arrays of MTZs to extend beyond the stratum corneum. It is this lasermicroporation that allows greater drug diffusion and delivery through these newly formed channels to areas beyond and surrounding the stratum corneum. ${ }^{12}$ Areas of unabated skin and follicular units allow for rapid re-epithelialization and remodeling of these MTZs; thus lowering the risk of posttreatment scarring. The depth and width of the micropores can be modulated to control the rate of drug delivery into the skin. ${ }^{11}$ As additional topical medications are recognized as potential combination therapy candidates, more clinical and esthetic applications for laser-assisted drug delivery are being uncovered. ${ }^{9}$ The complications and contraindications remain the same as those seen with the ablative fractionated laser and the particular drug being applied.

An animal study evaluating drug delivery by AFL assistance was conducted in Yorkshire swine treated with an AFL followed by topical application of methyl-5aminolevulinate (MAL), placebo cream, or no drug. ${ }^{14}$ The same topicals were applied to non-treated areas as controls, with all samples measured under fluorescent microscope. This study saw no porphyrin fluorescence in placebo cream or untreated skin areas. However, areas receiving AFL followed by MAL application had significantly higher porphyrin fluorescence of the epidermis, hair follicles, and dermis compared to MAL application alone. ${ }^{14}$ The success of this animal investigation in demonstrating the skin absorption enhancing effect that AFL can offer topical drug delivery clinically fostered a recent clinical study with photodynamic therapy (PDT). In this investigation, the goal was to discern if PDT efficacy in treating actinic keratoses may be improved with the assistance of AFL prior to PDT-MAL application 
compared with conventional PDT-MAL use alone. ${ }^{15}$ In this trial, 15 patients with actinic keratoses ranging in severity from grades I-III on the face and scalp were randomly assigned to one treatment of either AFL-PDT or PDT alone. Protoporphyrin IX fluorescence levels were higher in the AFL-treated skin. AFL-PDT treatment was found to be significantly more effective than PDT alone for all grades of actinic keratoses. The combined AFL-PDT treatment was also found to result in significantly fewer new actinic keratoses lesions, as well as improved photoaging of skin. Other topical medications that may benefit from combination with AFL include those used for treating skin cancers, such as 5-fluorouracil (5-FU) and imiquimod; this has been explored with some promising results in ex vivo samples. ${ }^{16,17}$ A clinical case study examined the pre-treatment of one patient with Bowen's disease with an Er:YAG laser prior to use of topical 5-FU to test the efficacy of laser-assisted delivery of 5-FU. ${ }^{18}$ Three lesions were targeted for the Er:YAG laser pretreatment in this patient with multiple Bowen's disease lesions. The results of this case study observed areas treated with the Er:YAG laser followed by topical 5-FU demonstrated an accelerated clinical response and reduced treatment duration compared to those lesions treated with 5-FU alone. Another area of laser assisted drug delivery has been in the treatment of hypertrophic scars with corticosteroid. Topical and intralesional corticosteroids have been a standard treatment for hypertrophic and restrictive scar reduction. One investigation examined the use of AFLs immediately prior to topical triamcinolone acetonide application. ${ }^{19}$ In this study, 15 participants with various degrees of hypertrophic scars were treated with three to five therapy sessions of AFL and topical triamcinolone acetonide over 2 or 3 month intervals. Evaluation of images at baseline and 6 months after the final treatment saw improvement overall in the areas of texture, hypertrophy, and dyschromia thus demonstrating a promising alternative therapy for reducing difficult to treat hypertrophic scars.

An exciting area that is emerging within AFL/topical pharmaceuticals concerns genetic manipulation (gene therapy) with topical genetic modulators. Topical genetic modulators function by interceding at either the level of transcription through DNA interacting agents, such as small molecules or oligonucleotides, or at post-transcriptional targets with RNA interference for localized targets of the dermis and epidermis. These modulators have resulted in moderate success treating actinic keratosis, skin cancers, and other heritable diseases in both animal and human studies. ${ }^{20}$ Yet, due to the hydrophilic character and negative charge that these genetic modulators possess, their penetration through an intact skin barrier is limited without assistance. ${ }^{21}$ One research group has been investigating the delivery of topical nucleotides with promising findings. In one study, they explored the enhanced absorption of topical antisense oligonucleotides after Er:YAG laser treatment in nude mice. ${ }^{22}$ Delivery of the antisense oligonucleotides was monitored by expression of reporter genes coding for beta-galactosidase and green fluorescent protein (GFP). The Er:YAG laser treatment produced a significant increase in antisense oligonucleotides permeation compared to the topical control. As a result of the stratum corneum ablation, DNA expression demonstrated a broad distribution through the epidermis to the subcutis with GFP expression being 160-fold greater than that in intact skin. This same group also evaluated the efficacy of fractional Er:YAG and $\mathrm{CO}_{2}$ lasers separately on skin permeation using both small interfering RNA (siRNA) and plasmid DNA vectors in nude mice. ${ }^{23}$ Fluorescence and confocal images demonstrated increased fluorescence intensities and penetration depths of siRNA and plasmid delivery with both lasers. In comparison to siRNA permeation on intact skin, use of the Er:YAG and $\mathrm{CO}_{2}$ lasers saw an 11-fold and 12-fold increase in respective siRNA penetration. This shows that topical nucleotide permeation is greatly assisted by the use of laser ablation and could offer a paradigm for facilitating delivery of future topical genetic modulators. Studies have demonstrated this increased permeation by macromolecules and nucleotides is promoted by the cylindrical micro-channels formed from the AFL that increase the contacting surface area for these topical modulators, and thus improve the penetration into the dermal and epidermal targets. ${ }^{24}$ Further investigations have explored AFL-drug combinations such as topical methotrexate for psoriasis, ${ }^{24}$ delivery of therapeutic antibodies for local immunosuppression, ${ }^{25}$ enhancement of timolol permeation for infantile hemangioma treatment, ${ }^{26}$ and facilitating the penetration of nanoparticles or liposomes in pharmaceutical delivery systems. ${ }^{27}$ AFL-assisted drug delivery is a promising tool for the future of dermatology and further investigations are being conducted to uncover more clinical uses.

\section{PRP and lasers}

Platelet-rich plasma (PRP) has been utilized within orthopedics for a number of years due to its ability to aid bone, ligament, and tendon healing. Currently, it is being used for applications within dermatology. These healing properties are attributed to the increased concentrations of autologous growth factors and secretory proteins enhancing the healing process by the recruitment, proliferation, and 
differentiation of cells involved in tissue regeneration. ${ }^{28}$ Concentrations of at least $1,000,000$ platelets $/ \mu \mathrm{L}$ of plasma are associated with the enhancement of healing (normal platelet counts in the blood ranging between $150,000 / \mu \mathrm{L}$ to $350,000 / \mu \mathrm{L}){ }^{29} \mathrm{PRP}-$ enhanced healing is derived from this delivery of growth factors and cell signaling molecules such as TGF- $\beta$, PDGF, IGF-I, IGF-II, FGF, and VEGF in the stored platelet granules. ${ }^{30}$ Preparation of PRP begins by harvesting the recipient's own whole blood, using citrate to inhibit the clotting cascade, followed by a centrifugation which separates and concentrates the platelets. PRP is then activated by the addition of calcium chloride to initiate clot formation that releases the concentrated growth factors from the platelet granules. ${ }^{31}$ As an autologous treatment, few complications are seen with hypersensitivity or allergy. In the last decade, PRP has been employed in the treatment of skin graft sites, burns, scars, hair loss disorders, and various types of ulcers. PRP has been explored as an adjunct treatment for wrinkles, dyschromia, and photo damaged skin and used in tandem with other modalities, such as ablative laser resurfacing. ${ }^{32}$ In these investigations, activated PRP is applied topically or injected in a layer over the laser ablated treated skin in order to deliver the concentrated growth factors to enhance the healing and rejuvenation, as well as diminishing patient down time.

In reducing wrinkles, fractionated $\mathrm{CO}_{2}$ laser resurfacing has shown excellent efficacy but carries an increased risk for adverse effects such as scarring, prolonged erythema, and longer patient recovery. One study explored the use of PRP to observe its benefits on wound healing after fractionated $\mathrm{CO}_{2}$ laser post-treatment. ${ }^{33}$ This investigation was performed on a group of patients' bilateral inner arms, with one side receiving fractionated $\mathrm{CO}_{2}$ laser only while the other received fractionated $\mathrm{CO}_{2}$ laser with PRP. Evaluation of the patients after 28 days saw faster wound healing in areas treated with fractionated $\mathrm{CO}_{2}$ laser with PRP. Significantly faster recovery of transepidermal water loss (TEWL) was observed, and erythema and melanin indexes were lower on the PRP-treated side. Biopsies taken 4 weeks after the treatment with PRP demonstrated thicker collagen bundles with more advanced stages of wound healing compared with the control areas. These results suggest that the use of PRP after fractionated $\mathrm{CO}_{2}$ laser resurfacing has potential as a more effective method for enhancing wound healing and improving skin tightening than $\mathrm{CO}_{2}$ laser only.

Other studies have supported using a combination of fractionated $\mathrm{CO}_{2}$ laser resurfacing with PRP on individuals with facial acne and acne scars. ${ }^{34}$ One group performed a split-face trial on 14 participants with acne scars who received one session of fractional $\mathrm{CO}_{2}$ resurfacing followed by the treatment with PRP intradermal injections on one side of the face and normal saline intradermal injections on the other side of the face. Final evaluation of the participants saw their total duration of erythema, edema, and crusting to be shorter on the PRP-treated side in comparison to the control side. Moreover, 4 months post therapy, the overall degree of clinical improvement was significantly better on the PRP treated sides. A separate study performed a similar splitfacial study on acne scar reduction comparing fractionated $\mathrm{CO}_{2}$ laser resurfacing alone or combined with PRP. However, these investigators chose to examine the efficacy and safety of topical versus injected PRP administration. ${ }^{35}$ This study used patients with acne scars whom had all received fractionated $\mathrm{CO}_{2}$ laser first and were then randomly split into two treatment groups. One group received intradermal PRP on one facial side and intradermal saline on the other. The other group received intradermal PRP to one facial side and topical PRP to the other side. Each patient received 3 monthly treatments and all participants were evaluated 6 months after the final treatment. These studies demonstrated a better response, fewer side effects, and shorter downtime in acne scars treated with fractionated $\mathrm{CO}_{2}$ laser with PRP compared to laser alone. No significant differences in response or downtime were seen between areas receiving either topical or intradermal injected PRP treatment in this study. The use of the Er:YAG laser combined with PRP for facial acne scars or acne scars has also been reported with similar successful outcomes. ${ }^{36}$ A study involving 22 patients with facial acne scars received treatment with the Er:YAG laser and PRP. Despite moderate and mild transient erythema being observed after three treatments, almost all the participants were satisfied with their outcomes, and physician evaluation indicated greater than $50 \%$ improvement in the treated acne and acne scars. Though further investigation is needed into the combination therapy of fractionated laser and PRP beyond wound healing and acne scar reduction, PRP administration with both $\mathrm{CO}_{2}$ and erbium fractional lasers treatments does show promise with enhanced recovery and improved clinical appearance as an adjunct therapy to fractionated laser alone.

\section{Laser radiofrequency resurfacing}

Radiofrequency technology has been growing in use through the last decade and has recently been combined with laser ablation. Radiofrequency has become popular for its effectiveness in reducing rhytides and skin laxity by sparing the epidermis through a method that traditionally produces lower temperatures than lasers and can be focused specifically 
to the dermis. ${ }^{37}$ This method employs electromagnetic energy to oscillate charged particles within a target tissue zone to generate heat. ${ }^{38}$ The heat production results in the skin tightening within the dermal tissue from the immediate denaturation and contraction of collagen fibers, and later initiates new collagen formation in subsequent weeks and months. ${ }^{39}$ As this technology has advanced in the recent years, many radiofrequency systems have emerged from the original unipolar and bipolar devices to the newer multipolar, fractional, pulsed, and phase-controlled devices that are available today. Radiofrequency systems are suitable for the treatment of acne scars, keloids, rosacea, and inflammatory acne, as well as to improve skin laxity and reduce rhytides. ${ }^{38}$ Since there is no chromophore dependence with radiofrequency, melanin absorption is not an issue and allows radiofrequency as a treatment option for a greater range of skin types.

Combination therapy using the ablative laser with radiofrequency has been reported in recent years. One of the few published studies with this combination therapy examined the treatment of acne and traumatic scars using the Smartxide ${ }^{2}$ DOT/radio frequency (RF) device (DEKA, Calenzano, Italy). ${ }^{40}$ This device is unique as it has the $\mathrm{CO}_{2}$ laser and radiofrequency in one device, to produce epidermal coagulation for a resurfacing effect and dermal denaturation for deeper remodeling. Patients with severe acne scars and/or traumatic scars underwent two treatments with the Smartxide ${ }^{2}$ DOT/RF device scheduled 1 month apart. Evaluation using a global esthetic improvement scale saw $73.3 \%$ of patients' state much improved or greater immediately after their last treatment with $100 \%$ seeing improvement 12 months post-treatment. Moderate erythema and edema were seen with participants with darker skin types that receded within hours of treatment with all patients returning to their everyday activities after the treatment without delay. This ablative laser-radiofrequency combination therapy study established an emerging option in resurfacing treatments as demonstrated with significant post-treatment improvement in patients' severe acne and atrophic scars.

\section{Ablative radiofrequency}

Radiofrequency is also being used as a single modality for skin treatments. Fractioned radiofrequency has been recently described, and is also able to ablate the skin surface. ${ }^{38}$ The same principle applies to its delivery of electromagnetic energy to cause superficial dermal coagulation while still able to ablate the epidermis immediately around the electrodes of the device with the same energy. This fractionated radiofrequency results in epidermal and subepidermal ablation under the conductive pins that reproduces similar effects as a fractional $\mathrm{CO}_{2}$ laser with dermal heating, seen in the non-ablative lasers and devices. ${ }^{41}$ This combination of epidermal ablation and dermal heating with radiofrequency, called sublative resurfacing in some studies, is suitable for skin types I-IV, for the treatment of skin laxity, wrinkles, enlarged pores, pigmented lesions, acne, telangiectasias, and scaring from trauma or acne. Patient recovery and down time periods are significantly lower when compared with ablative laser healing times, with minimal adverse effects. ${ }^{42}$

An initial study evaluating the clinical efficacy of the Matrix RF (Syneron, Irvine, CA USA) fractional ablative radiofrequency device was performed on two groups to monitor its histological effects on wound healing, and its effectiveness for the treatment of wrinkles. ${ }^{41}$ The first group of participants who were scheduled for abdominoplasty received fractional RF treatments to their abdominal area and later had biopsies performed following their abdominoplasty. The second group of participants received three facial treatments at 3-4 week intervals with treatment responses evaluated by the participants and physicians. Histological analysis performed on the abdominoplasty samples demonstrated complete tissue healing 2 days post-treatment, with well-organized, denser collagen deposition. A majority of participants receiving the facial treatments were satisfied with the treatment results and noted minimal pain, no permanent side effects, or significant downtime. After 1 month, the physicians' assessments observed an overall improvement in greater than $80 \%$ of participants in the facial parameters of brightness, skin tightness, and wrinkles.

Another investigation examined the effectiveness of the Fractora (Invasix, Irvine, CA USA) ablative radiofrequency device in treating skin laxity, fine and course wrinkles, and ability to improve skin texture and tone. ${ }^{42}$ This was a multiple center study with subjects of Caucasian skin types I-II and Asian skin type III-IV. These subjects received a single full-face treatment using the Fractora hand piece at energies of 50-62 mJ/pin applied for skin types I-III and 10-40 mJ/pin for skin types IV. Biopsies were also taken and histological analysis demonstrated an initiation of neocollagenesis and tightening of existing collagen fibrils similar to that seen with the ablative $\mathrm{CO}_{2}$ laser. Physician and subject evaluation of the Fractora treatment revealed overall enhancement of skin quality after 3-6 months with significant improvements in wrinkles, laxity, pigmentation, skin tightening, and acne scars in all skin types. Most subjects in the study experienced some edema and erythema for the first 7 days post-treatment 
with resolution after 2 weeks. Re-epithelialization of the epidermis was seen within 4-7 days with no observed cases of delayed healing or adverse reactions. A third ablative radiofrequency device, the EndyMed PRO (EndyMed Medical, Caesarea, Israel), has likewise demonstrated effective treatment in reducing wrinkles and skin laxity in a clinical study. ${ }^{43}$ Participants with Fitzpatrick's skin type II-III ranging between the Fitzpatrick wrinkle and elastosis scale of 5-8 underwent treatment with the EndyMed PRO device once a month for 3 months on the face. Evaluation of the patient treatment outcomes were assessed by the study's dermatologists 4 weeks after the last treatment when patient pain and satisfaction levels were scored with questionnaires. Patients reported minimal or no pain after treatment and all patients showed significant reduction in the Fitzpatrick wrinkle score, dropping from initial average baseline of 7.3 to 4.13 months after the third treatment. Together, these clinical assessments and histological findings reveal that ablative radiofrequency treatments are safe, minimally painful, and effective at improving skin texture, laxity, and wrinkles in various skin types. Consideration should be given to ablative radiofrequency as an alternative to ablative laser skin resurfacing.

\section{Plasma resurfacing}

An emerging method utilizing the state of matter known as plasma to create a thermal effect on the skin through the use of positively ionized gasses is plasma skin resurfacing (PSR). As this plasma comes in contact with the resurfacing target, the positive ions capture back electrons while energy is released. With PSR, a gaseous nitrogen source is used to form a plasma that releases thermal energy that eliminates oxygen from the targeted skin surface. ${ }^{44,45}$ PSR is not dependent on a chromophore for its use and does not vaporize tissue as ablative lasers do. This resurfacing modality has been hypothesized to function by forming a layer of desiccated epidermis creating a natural barrier that facilitates accelerated healing with generation of new epidermis. PSR also penetrates to the upper dermis resulting in thermal denaturation of surrounding collagen, thereby increasing fibroblast activity, which has been shown to continue up to a year after the plasma treatments. ${ }^{46}$ Plasma resurfacing has been safely used in Fitzpatrick skin types I-IV. It has been approved for the treatment of many skin conditions including photoaging, acne scars, rhytides, dyschromias, skin laxity, as well as the treatment of actinic keratosis and seborrheic keratosis. In contrast to the ablative laser, plasma resurfacing treatments have a very low incidence of side effects such as permanent hypopigmentation, scaring, or prolonged erythema. ${ }^{47}$

An early study using the Portrait PSR (Rhytec, Waltham, MA USA) device examined its effects both clinically and histologically following multiple single-pass treatments for full-facial resurfacing. ${ }^{48}$ Participants received three full-face treatments at 3 week intervals with evaluation of epidermal quality, downtime, and erythema performed prior to each resurfacing treatment as well as 4 days post-treatment. Participant assessments were also performed at 1 and 3 months after the final treatment. Evaluation of subjects noted complete re-epithelialization 4 days after therapy with lingering erythema persisting 6 days following treatment. Two-thirds of participants experienced an improvement in their overall facial appearance and one-third of participants noted a reduction in their facial wrinkles. Assessment of the biopsies revealed new collagen formation at the epidermaldermal junction 3 months after treatment. No scarring or permanent pigmentation changes were seen during or after the study. A separate 3 month investigation conducted on nonfacial areas in ten patients examined the efficacy of plasma resurfacing on photodamaged skin of the neck, chest, and hands. They received only a single-pass with the Portrait PSR system. All sites showed a significant reduction in wrinkle severity and hyperpigmentation, as well as improved skin texture. Though treatments using higher-energy settings resulted in greater benefits in patients' skin, they also prolonged their healing in these areas. Comparison of biopsies taken before and 90 days post-treatment, revealed increased epidermal thickening, reduction of solar elastosis, and new collagen deposition in all biopsy specimens.

Acne scarring is a common and difficult to treat condition. With the success of PSR as an effective treatment for facial rhytides and benign skin lesions, a group investigated the safety and efficacy of PSR for the treatment of acne scarring. ${ }^{49}$ In their study, patient skin types I-III with acne scarring underwent a single PSR treatment with two high-energy passes. Both patient and physician ratings demonstrated around a one-third improvement in the treated areas after 6 months. After 4-6 days, most patients saw complete re-epithelialization, with no serious adverse events encountered. Reduction of traumatic scars by PSR is also being explored. In a group of Asian patients, an investigation examined the effectiveness of plasma treatment for traumatic scars that were treated monthly for 3 months. ${ }^{50}$ Almost half of the subjects experienced a greater than 50\% improvement in their traumatic scars with the average re-epithelization of the treated skin being 5-9 days. Deep traumatic scars in this subject group 
were noted to be resistant to plasma treatment. Together, all patients tolerated the treatments with minimal pain, with only temporary localized hyperpigmentation observed in a few patients which resolved in 3 months. Another investigation into scar reduction using PSR by the same group examined the effectiveness in treating mesh skin-grafted scars. ${ }^{51}$ In this small study, four patients with mesh skin-grafted scars were treated at monthly intervals with plasma resurfacing. They were seen 1 week after each individual treatment as well as 3 months after the final treatment. Analysis from the physician assessment of images and patient questionnaires noted that all four patients demonstrated a greater than $50 \%$ improvement in their mesh skin grafted scars with only temporary, localized hypopigmentation observed in two patients. These four patients showed improvement and tolerated the treatments well, which suggests that plasma treatment is an effective tool for the treatment of mesh skin-grafted scars.

PSR is a distinct resurfacing treatment with the ability to keep a layer of intact desiccated epidermis, which acts as a natural biologic dressing and promotes rapid healing. More studies need to be pursued to define important parameters, including the effect of multiple treatments, optimal treatment settings, and the duration of clinical results. PSR has the potential to be a viable alternative to ablative laser resurfacing.

\section{Microneedling}

Microneedling, also known as skin needling or percutaneous collagen induction therapy, is a procedure for skin resurfacing that involves the use of fine needles to create micro dermal wounds. ${ }^{52}$ These controlled micro skin injuries stimulate remodeling of existing collagen and promote formation of new collagen, elastin, and vascularization in the papillary dermis, which results in the reduction in appearance of fine lines and wrinkles, skin laxity, and scarring. ${ }^{53}$ Microneedling had its beginnings during the mid-1990s where multiple groups noted improvement after microneedle use on scars and wrinkles, such as Camirand et al observing scar improvement after treatment with a tattoo gun. ${ }^{54} \mathrm{~A}$ decade later, the rolling microneedle device was developed. Microneedling has become increasingly popular within clinical practices with brands such as the Dermaroller (Dermaroller, Friesenheim, France) and Dermapen (Equipmed Pty Ltd, North Ryde, Australia). ${ }^{55,56}$ This therapy is considered a safe skin resurfacing therapy due to minimal damage induced to the skin, resulting in much less epidermal injury than seen with laser ablation. There is also a lower risk of hyperpigmentation and scarring with microneedling, making it a suitable treatment choice for persons with thin, sensitive, or ethnic skin types (skin types $>$ III).

Recent studies have demonstrated success using microneedling treatments to improve scar appearance and reduce wrinkles. One study examined the efficacy of microneedling in acne scars. ${ }^{53}$ In this study, rolling acne scars were treated with two sessions using the Dermaroller. Analysis performed 8 weeks after the final treatment session noted all patients experiencing an observable reduction in their surface irregularity and an overall improvement of the rolling scar severity. A separate study evaluated the microneedling treatment on atrophic facial scars. ${ }^{57}$ Patients with atrophic facial scarring were given multiple Dermaroller treatments and assessed 2 months after their final treatment. Almost $95 \%$ of subjects noted a reduction in the severity of their scars by 1 or 2 grades and a majority of the subjects assessed their treatment as "excellent" on a 10-point scale. Another investigation has seen success with microneedling reducing wrinkles and aging around the neck region. ${ }^{58}$ In this study, patients were treated with two sessions of microneedling with assessments taken before and 32 weeks after the final treatment. Final analysis in this study revealed that almost $90 \%$ of the patients' neck regions showed improvement in their wrinkles with a reduction in skin surface irregularity. The effectiveness of microneedling therapy in treating female patients with striae distensae alba or rubra has also been examined. ${ }^{59}$ These patients received three treatments at 4 week intervals using a disk microneedle therapy system (DTS roller; DTS-MG, Inc., Seoul, South Korea). Analysis 3 months after the final treatment saw clear improvement in $43.8 \%$ of patients' striae distensae with $>85 \%$ of patients being somewhat or highly satisfied with their treatment results. Together, these studies confirm that fractionated injury from skin needling has an immediate effect on improving multiple scars types as well as wrinkles and may be an effective treatment option in their management.

Microneedles are also being integrated with transdermal drug administration. Similar to laser-assisted drug delivery, the microneedles penetrate through the stratum corneum of the epidermis and allow greater delivery of topical medications through the skin layers. ${ }^{60}$ The design and composition of these drug-assisting microneedles have been classified into four categories: solid microneedles used to pretreat the skin, drug-coated microneedles, dissolving or biodegradable microneedles, and hollow microneedles. ${ }^{20}$ Several ongoing clinical trials are examining the use of microneedles and drug delivery, which could provide future therapies to treat 
multiple skin conditions and provide greater efficacy with minimal side effects.

\section{Crystal-free microdermabrasion}

Since its inception in Italy in the mid-1980s, microdermabrasion has remained a popular method for skin resurfacing and rejuvenation. This modality effectively exfoliates the outer layers of the epidermis using the propulsion of a mechanical medium, such as aluminum oxide microcrystals, at the skin through a handpiece. ${ }^{61}$ As the skin is exfoliated, the handpiece suctions the skin debris and microcrystals away through an accompanying vacuum. This modality has been used to treat light scarring, discolored and photodamaged skin, enlarged pores, and has been helpful in reducing the appearance of stretch marks and fine lines. ${ }^{62}$ This method also stimulates papillary dermis thickening. Despite its effectiveness, microdermabrasion is not recommended for all skin types and is used cautiously in persons with Fitzpatrick skin types IV-VI, rosacea, sensitive or thin skin because of the increased risk of irritation and hyperpigmentation. ${ }^{63}$ Though complications of microdermabrasion are minimal, patients' largest complaints post-procedure are temporary dryness and photosensitivity. ${ }^{64}$ Newer methods of microdermabrasion have moved toward microcrystal-free modalities exemplified by diamond tip microdermabrasion and hydradermabrasion.

The diamond tip microdermabrasion system is a handset with a contact point composed of diamond fragments that are adjustable for size and abrasiveness enabling treatment of a large variety of skin types and thicknesses. ${ }^{65}$ This method functions with a polishing motion to wear down the epidermal layers while the vacuum suctions away the debris and dead cells. Treatment is reliant on the operator's skill with the handpiece and the manual pressure applied to the epidermis along with the level of suction being used. ${ }^{66}$ Advantages of the diamond tip can be seen with treatments on areas around the eyes and mouth that can be treated without risk of microcrystals causing irritation, damage, or being ingested. Also, the tips are reusable reducing the cost of consumable microcrystals, with treatment times being shorter due to more effective clearance of debris and dead cells. The indications and contraindications for use of the diamond tip are the same as with traditional microdermabrasion. One study conducted using either a coarse-grit or medium-grit abrasiveness diamond handpiece was performed on a group of adults with sun-damaged skin on their arms. ${ }^{67}$ In this study, the coarse-grit handpiece resulted in an increased production of a wide variety of compounds associated with wound healing and skin remodeling like cytokeratin 16, antimicrobial peptides, matrix metalloproteinases, and collagen precursors. Another study investigating skin barrier changes post diamond microdermabrasion examined changes in TEWL, hydration, and erythema following diamond microdermabrasion. ${ }^{68}$ This group treated with the diamond microdermabrasion observed a significant increase in TEWL immediately after and at 24 hours after the procedure in comparison to an untreated skin control. Increased hydration and erythema were immediately seen following the microdermabrasion with return to baseline 24 hours post-treatment. Overall, their results show that skin barrier function recovers within 48 hours of diamond microdermabrasion.

The second developing method is hydradermabrasion. It works in the same manner as microdermabrasion but instead of exfoliating with propelled microcrystals, hydradermabrasion uses a combination of oxygen and aqueous solutions at supersonic speeds to remove debris and dead cells that are then suctioned away. ${ }^{69,70}$ This new modality is novel because as the treatment is exfoliating the epidermis, it is also hydrating the skin within the same treatment pass. This supersonic microdroplet jet results in a pressured widening of micro-canals in the outer skin layers that facilitate greater hydration and cleansing of the skin. This moisturizing component allows for less irritation, reduced discomfort, and quicker recovery. Solutions and serums can also be formulated allowing better cleansing, extraction, and exfoliation while possessing antioxidant properties targeting specific skin types, textures, and conditions which cannot be delivered with traditional microdermabrasion. ${ }^{71,72}$ Hydradermabrasion has the same indications for use as microdermabrasion making it an excellent choice for persons with darker skin tones, aging skin, sensitive skin areas, oily, and dry skin complexions. A study on the efficacy of hydradermabrasion for facial rejuvenation was conducted comparing volunteers who received hydradermabrasion facial treatments using an antioxidant serum or who just received the same antioxidant serum manually applied to the skin. ${ }^{73}$ Six treatments were conducted on a 7-10 day interval to determine whether antioxidant levels could be increased in the skin and skin quality improved with this technique. Results of this study demonstrated that hydradermabraded skin possessed increased epidermal and papillary dermal thickness as well as greater antioxidant levels. Further histological examination saw a replacement of elastic dermal tissue, collagen hyalinization, and fibroblast density correlating with a decrease in the appearance of fine lines, pore size, and hyperpigmentation in hydradermabrasion treated areas 6 weeks post-treatment with no patient complications. With its ability to add hydration, oxygen, and antioxidants to the 
exfoliated skin, this study demonstrates that hydradermabrasion treatment is effective at improving skin quality and should be considered an alternative to microdermabrasion.

\section{Resurfacing as a cancer prophylaxis}

The incidence of non-melanoma skin cancers (NMSC) rises with age with more than $80 \%$ of the total number of skin cancers appearing in individuals after the age of $50 .{ }^{74}$ Prevention of these skin cancers before they develop is important not only due to these increasing skin cancer numbers, but also because of the large financial cost of treatment and potential disfiguration associated with these types of neoplasia. Current treatments such as cryotherapy, electrosurgery, curettage, PDT, and topical medicines like 5-FU or imiquimod work through physical, chemical or immune-mediated destruction of established precancerous or low-grade lesions. ${ }^{75}$ These treatments focus only on the precancerous and tumor cells and do not address the underlying processes that cause aged skin to be more susceptible to neoplasia. Recently, the increased susceptibility of elderly patients to developing NMSC was described to be due in part to the failure of geriatric skin to respond appropriately to ultraviolet B (UVB) irradiation found in sunlight. ${ }^{76}$ This inappropriate UVB response represents a failure of the protective properties found in young skin that halts the propagation of UVB-induced mutations in basal layer keratinocytes. ${ }^{77,78}$ The appropriate protective UVB response in young skin is mediated by insulin-like growth factor-1 (IGF-1) secreted from dermal fibroblasts. Dermal IGF-1 production is necessary to activate the IGF-1 receptor present on basal keratinocytes in order to preserve this appropriate UVB response. This UVB response is deficient in geriatric skin due to an increasing proportion of senescent dermal fibroblasts resulting in a silencing of dermal IGF-1 production. It is this inappropriate UVB response observed in geriatric skin that allows DNA mutations to propagate in proliferating basal keratinocytes, thus initiating photocarcinogenesis. ${ }^{77,78}$ Importantly, restoration of dermal IGF-1 expression could prevent the initiation of UVB-induced carcinogenesis by restoring this secretion of dermal IGF-1. In fact, resurfacing techniques such as dermabrasion and fractionated laser resurfacing will normalize IGF-1 expression in geriatric skin and re-establish the youthful, appropriate UVB response. ${ }^{79,80}$ These treatment modalities are well known for their cosmetic applications in part by restoring dermal collagen expression through the removal of senescent fibroblasts and recruitment of new active fibroblasts, but these treatments also result in increased levels of dermal IGF-1 expression in the skin. Studies are currently in progress to examine the long-term effectiveness of these resurfacing methods on numbers of senescent fibroblasts and dermal IGF-1 production after 1 and 2 years post-resurfacing, as well as identifying other resurfacing techniques that restore the appropriate UVB response. Together, these results show that resurfacing techniques possess applications beyond cosmetic purposes and show potential promise as protective therapies for geriatric individuals against future actinic neoplasia.

\section{Conclusion}

The development of technological advancements and new treatment methodologies within skin resurfacing has brought about several emerging therapies. Many of these new therapies demonstrate promising results that closely approach the efficacy of the ablative laser in clinical outcomes, while further minimizing downtime and side effects. The laser combination/adjunct therapies, radiofrequency ablation, PSR, microneedling, and other crystal-free microdermabrasion methods discussed here are evolving into practice today and have the potential to become routine resurfacing therapies in the future. As the demand continues to grow, more research is needed to develop the next innovative resurfacing treatments that continue to improve upon efficacy and safety.

\section{Acknowledgments}

DFS and JBT are supported by grants from the National Institute of Environmental Health Sciences (R01ES020866) and the US Department of Veterans Affairs (1101CS000809). MML is supported by a grant from the National Institutes of Health (T32R062495).

\section{Disclosure}

The authors have no conflicts of interest in this work.

\section{References}

1. ASDS [homepage on the Internet]. ASDS survey: Skin cancer, cosmetic procedures jump 22 percent in 2013. 2014. Available from: https://www. asds.net/_Media.aspx?id=7744. Accessed February 10, 2014.

2. Lipozencic J, Bukvic Mokos Z. Dermatologic lasers in the treatment of aging skin. Acta Dermatovenerol Croat. 2010;18(3):176-180.

3. Alster TS, Lupton JR. Lasers in dermatology. An overview of types and indications. Am J Clin Dermatol. 2001;2(5):291-303.

4. Airan LE, Hruza G. Current lasers in skin resurfacing. Facial Plast Surg Clin North Am. 2005;13(1):127-139.

5. Tanzi EL, Lupton JR, Alster TS. Lasers in dermatology: four decades of progress. J Am Acad Dermatol. 2003;49(1):1-31.

6. Smith KC, Schachter GD. YSGG 2790-nm Superficial Ablative and Fractional Ablative Laser Treatment. Facial Plast Surg Clin North Am. 2011;19(2):253-260

7. Stulhofer Buzina D, Lipozencic J, Bukvic Mokos Z, Ceovic R, Kostovic K Ablative laser resurfacing: is it still the gold standard for facial rejuvenation? Acta dermatovenerol Croat. 2010;18(3):190-194.

8. Alexiades-Armenakas MR, Dover JS, Arndt KA. Fractional laser skin resurfacing. J Drugs Dermatol. 2012;11(11):1274-1287. 
9. Saedi N, Jalian HR, Petelin A, Zachary C. Fractionation: past, present, future. Semin Cutan Med Surg. 2012;31(2):105-109.

10. Saedi N, Petelin A, Zachary C. Fractionation: a new era in laser resurfacing. Clin Plast Surg. 2011;38(3):449-461.

11. Bloom BS, Brauer JA, Geronemus RG. Ablative fractional resurfacing in topical drug delivery: an update and outlook. Dermatol Surg. 2013;39(6):839-848.

12. Hædersdal M, Sakamoto FH, Farinelli WA, Doukas AG, Tam J, Anderson RR. Fractional CO2 laser-assisted drug delivery. Lasers Surg Med. 2010;42(2):113-122.

13. Prasanthi D, Lakshmi PK. Vesicles - mechanism of transdermal permeation: a review. Asian Journal of Pharmaceutical and Clinical Research. 2012;5(1):18-25.

14. Haak CS, Farinelli WA, Tam J, Doukas AG, Anderson RR, Haedersdal M. Fractional laser-assisted delivery of methyl aminolevulinate: Impact of laser channel depth and incubation time. Lasers Surg Med. 2012;44(10):787-795.

15. Togsverd-Bo K, Haak CS, Thaysen-Petersen D, Wulf HC, Anderson RR, Haedersdal M. Intensified photodynamic therapy of actinic keratoses with fractional $\mathrm{CO} 2$ laser: a randomized clinical trial. $\mathrm{Br} J$ Dermatol. 2012;166(6):1262-1269.

16. Lee WR, Shen SC, Wang KH, Hu CH, Fang JY. The effect of laser treatment on skin to enhance and control transdermal delivery of 5-fluorouracil. J Pharm Sci. 2002;91(7):1613-1626.

17. Lee WR, Shen SC, Al-Suwayeh SA, Yang HH, Yuan CY, Fang JY. Laser-assisted topical drug delivery by using a low-fluence fractional laser: imiquimod and macromolecules. J Control Release. 2011;153(3): 240-248.

18. Wang KH, Fang JY, Hu CH, Lee WR. Erbium:YAG laser pretreatment accelerates the response of Bowen's disease treated by topical 5-fluorouracil. Dermatol Surg. 2004;30(3):441-445.

19. Waibel JS, Wulkan AJ, Shumaker PR. Treatment of hypertrophic scars using laser and laser assisted corticosteroid delivery. Lasers Surg Med. 2013;45(3):135-140.

20. Gratieri T, Alberti I, Lapteva M, Kalia YN. Next generation intra- and transdermal therapeutic systems: using non- and minimally-invasive technologies to increase drug delivery into and across the skin. Eur $J$ Pharm Sci. 2013;50(5):609-622.

21. Lee WR, Shen SC, Zhuo RZ, Wang KC, Fang JY. Enhancement of topical small interfering RNA delivery and expression by low-fluence erbium:YAG laser pretreatment of skin. Hum Gene Ther. 2009;20(6):580-588.

22. Lee WR, Shen SC, Liu CR, Fang CL, Hu CH, Fang JY. Erbium:YAG laser-mediated oligonucleotide and DNA delivery via the skin: an animal study. J Control Release. 2006;115(3):344-353.

23. Lee WR, Shen SC, Chen WY, Aljuffali IA, Suen SY, Fang JY. Noninvasive delivery of siRNA and plasmid DNA into skin by fractional ablation: Erbium:YAG laser versus CO laser. Eur J Pharm Biopharm. 2014;86(3):315-323.

24. Lee WR, Shen SC, Fang CL, Zhuo RZ, Fang JY. Topical delivery of methotrexate via skin pretreated with physical enhancement techniques: low-fluence erbium:YAG laser and electroporation. Lasers Surg Med. 2008;40(7):468-476.

25. Yu J, Kalaria DR, Kalia YN. Erbium:YAG fractional laser ablation for the percutaneous delivery of intact functional therapeutic antibodies. $J$ Control Release. 2011;156(1):53-59.

26. Ma G, Wu P, Lin X, et al. Fractional Carbon Dioxide Laser-Assisted Drug Delivery of Topical Timolol Solution for the Treatment of Deep Infantile Hemangioma: A Pilot Study. Pediatr Dermatol. 2014;31(3): 286-291.

27. Genina EA, Bashkatov AN, Dolotov LE, et al. Transcutaneous delivery of micro- and nanoparticles with laser microporation. J Biomed Opt. 2013;18(11):111406.

28. Foster TE, Puskas BL, Mandelbaum BR, Gerhardt MB, Rodeo SA. Platelet-rich plasma: from basic science to clinical applications. Am J Sports Med. 2009;37(11):2259-2272.

29. Marx RE. Platelet-Rich Plasma (PRP): What Is PRP and What Is Not PRP? Implant Dent. 2001;10(4):225-228.
30. Kim DH, Je YJ, Kim CD, et al. Can Platelet-rich Plasma Be Used for Skin Rejuvenation? Evaluation of Effects of Platelet-rich Plasma on Human Dermal Fibroblast. Ann Dermatol. 2011;23(4):424-431.

31. Akhundov K, Pietramaggiori G, Waselle L, et al. Development of a cost-effective method for platelet-rich plasma (PRP) preparation for topical wound healing. Ann Burns Fire Disasters. 2012;25(4): 207-213

32. Arshdeep, Kumaran MS. Platelet-rich plasma in dermatology: Boon or a bane? Indian J Dermatol Venereol Leprol. 2014;80(1):5-14.

33. Na JI, Choi JW, Choi HR, et al. Rapid healing and reduced erythema after ablative fractional carbon dioxide laser resurfacing combined with the application of autologous platelet-rich plasma. Dermatol Surg. 2011;37(4):463-468.

34. Lee JW, Kim BJ, Kim MN, Mun SK. The efficacy of autologous platelet rich plasma combined with ablative carbon dioxide fractional resurfacing for acne scars: a simultaneous split-face trial. Dermatol Surg. 2011;37(7):931-938.

35. Gawdat HI, Hegazy RA, Fawzy MM, Fathy M. Autologous platelet rich plasma: topical versus intradermal after fractional ablative carbon dioxide laser treatment of atrophic acne scars. Dermatol Surg. 2014;40(2): $152-161$.

36. Zhu JT, Xuan M, Zhang YN, et al. The efficacy of autologous plateletrich plasma combined with erbium fractional laser therapy for facial acne scars or acne. Mol Med Rep. 2013;8(1):233-237.

37. Reddy BY, Hantash BM. Emerging technologies in aesthetic medicine. Dermatol Clin. 2009;27(4):521-527.

38. Krueger N, Sadick NS. New-generation radiofrequency technology. Cutis. 2013;91(1):39-46.

39. Zelickson BD, Kist D, Bernstein E, et al. Histological and ultrastructural evaluation of the effects of a radiofrequency-based nonablative dermal remodeling device: a pilot study. Arch Dermatol. 2004;140(2): 204-209.

40. Tenna S, Cogliandro A, Piombino L, Filoni A, Persichetti P. Combined use of fractional $\mathrm{CO} 2$ laser and radiofrequency waves to treat acne scars: a pilot study on 15 patients. J Cosmet Laser Ther. 2012;14(4): 166-171.

41. Hruza G, Taub AF, Collier SL, Mulholland SR. Skin rejuvenation and wrinkle reduction using a fractional radiofrequency system. $J$ Drugs Dermatol. 2009;8(3):259-265.

42. Mulholland RS, Ahn DH, Kreindel M, Paul M. Fractional Ablative Radio-Frequency Resurfacing in Asian and Caucasian Skin: A Novel Method for Deep Radiofrequency Fractional Skin Rejuvenation. Journal of Cosmetics, Dermatological Sciences and Applications. 2012;2(3): 144-150.

43. Dahan S, Rousseaux I, Cartier H. Multisource radiofrequency for fractional skin resurfacing-significant reduction of wrinkles. $J$ Cosmet Laser Ther. 2013;15(2):91-97.

44. Alster TS, Konda S. Plasma Skin Resurfacing for Regeneration of Neck, Chest, and Hands: Investigation of a Novel Device. Dermatol Surg. 2007;33(11):1315-1321.

45. Heinlin J, Isbary G, Stolz W, et al. Plasma applications in medicine with a special focus on dermatology. J Eur Acad Dermatol Venereol. 2011;25(1):1-11.

46. Lin MG, Yang TL, Chiang CT, et al. Evaluation of dermal thermal damage by multiphoton autofluorescence and second-harmonic-generation microscopy. J Biomed Opt. 2006;11(6):064006.

47. Foster KW, Moy RL, Fincher EF. Advances in plasma skin regeneration. J Cosmet Dermatol. 2008;7(3):169-179.

48. Bogle MA, Arndt KA, Dover JS. Evaluation of plasma skin regeneration technology in low-energy full-facial rejuvenation. Arch Dermatol. 2007;143(2):168-174.

49. Gonzalez MJ, Sturgill WH, Ross EV, Uebelhoer NS. Treatment of acne scars using the plasma skin regeneration (PSR) system. Lasers Surg Med. 2008;40(2):124-127.

50. Kono T, Groff WF, Sakurai H, Yamaki T, Soejima K, Nozaki M. Treatment of traumatic scars using plasma skin regeneration (PSR) system. Lasers Surg Med. 2009;41(2):128-130. 
51. Higashimori T, Kono T, Sakurai H, Nakazawa H, Groff WF. Treatment of mesh skin grafted scars using a plasma skin regeneration system. Plast Surg Int. 2010;2010:874348.

52. Fife D. Practical evaluation and management of atrophic acne scars tips for the general dermatologist. J Clin Aesthet Dermatol. 2011;4(8): 50-57.

53. Fabbrocini G, Fardella N, Monfrecola A, Proietti I, Innocenzi D. Acne scarring treatment using skin needling. Clin Exp Dermatol. 2009;34(8):874-879.

54. Camirand A, Doucet J. Needle dermabrasion. Aesthetic Plast Surg. 1997;21(1):48-51.

55. Doddaballapur S. Microneedling with dermaroller. J Cutan Aesthet Surg. 2009;2(2):110-111.

56. Fernandes D. Minimally invasive percutaneous collagen induction. Oral Maxillofac Surg Clin North Am. 2005;17(1):51-63.

57. Majid I. Microneedling therapy in atrophic facial scars: an objective assessment. J Cutan Aesthet Surg. 2009;2(1):26-30.

58. Fabbrocini G, De Vita V, Di Costanzo L, et al. Skin needling in the treatment of the aging neck. Skinmed. 2011;9(6):347-351.

59. Park KY, Kim HK, Kim SE, Kim BJ, Kim MN. Treatment of striae distensae using needling therapy: a pilot study. Dermatol Surg. 2012;38(11):1823-1828.

60. Bariya SH, Gohel MC, Mehta TA, Sharma OP. Microneedles: An emerging transdermal drug delivery system. J Pharm Pharmacol. 2012;64(1):11-29.

61. Kirkland EB, Gladstone HB, Hantash BM. What's new in skin resurfacing and rejuvenation? G Ital Dermatol Venereol. 2010;145(5):583-596.

62. Grimes PE. Microdermabrasion. Dermatol Surg. 2005;31(9 Pt 2): 1160-1165.

63. Shpall R, Beddingfield FC 3rd, Watson D, Lask GP. Microdermabrasion: a review. Facial Plast Surg. 2004;20(1):47-50.

64. Tan MH, Spencer JM, Pires LM, Ajmeri J, Skover G. The evaluation of aluminum oxide crystal microdermabrasion for photodamage. Dermatol Surg. 2001;27(11):943-949.

65. Ignon R, Nicolas EF, inventors; Edge Systems Corporation, assignee Microdermabrasion apparatus and method. United States patent US20130102978 A1. April 25, 2013.

66. Waldron SH, inventor; Altair Instruments, Inc., assignee. Microdermabrasion device and method of treating the skin surface. United States patent US 6241739 B1. June 5, 2001.
67. Karimipour DJ, Rittie L, Hammerberg C, et al. Molecular analysis of aggressive microdermabrasion in photoaged skin. Arch Dermatol. 2009;145(10):1114-1122.

68. Kim HS, Lim SH, Song JY, et al. Skin barrier function recovery after diamond microdermabrasion. J Dermatol. 2009;36(10):529-533.

69. Khalaj B, inventor; Khalaj Ben M, assignee. Hydro-dermabrasion apparatus and method of use. United States patent US 20070239173 A1. October 11, 2007.

70. Rosso L, inventor; L.I.C.A. S.R.L., assignee. Device for making hydromicroabrasions on human tissue. United States patent US 6432114 B1. August 13, 2002.

71. Waldron SH, Kent P, inventors; Altair Instruments, Inc., assignee. Device for applying liquid skincare products in combination with vacuum and abrasion. United States patent US 8221437 B2. July 17, 2012.

72. Burke KE. Photodamage of the skin: protection and reversal with topical antioxidants. J Cosmetic Dermatol. 2004;3(3):149-155.

73. Freedman BM. Hydradermabrasion: an innovative modality for nonablative facial rejuvenation. J Cosmet Dermatol. 2008;7(4): 275-280.

74. Kraemer KH. Sunlight and skin cancer: Another link revealed. Proc Nat Acad Sci U S A. 1997;94(1):11-14.

75. Travers JB, Spandau DF, Lewis DA, et al. Fibroblast senescence and squamous cell carcinoma: how wounding therapies could be protective. Dermatol Surg. 2013;39(7):967-973.

76. Lewis DA, Travers JB, Somani A-K, Spandau DF. The IGF-1/IGF-1R signaling axis in the skin: a new role for the dermis in aging-associated skin cancer. Oncogene. 2010;29(10):1475-1485.

77. Lewis DA, Travers JB, Spandau DF. A new paradigm for the role of aging in the development of skin cancer. J Invest Dermatol. 2009; 129(3):787-791.

78. Lewis D, Travers J, Spandau D. Aging-associated Non-melanoma Skin Cancer: A Role for the Dermis. In: Farage M, Miller K, Maibach H, editors. Textbook of Aging Skin. Berlin Heidelberg: Springer; 2010:587-599.

79. Lewis DA, Travers JB, Machado C, Somani AK, Spandau DF. Reversing the aging stromal phenotype prevents carcinoma initiation. Aging (Albany NY). 2011;3(4):407-416.

80. Spandau DF, Lewis DA, Somani AK, Travers JB. Fractionated laser resurfacing corrects the inappropriate UVB response in geriatric skin. J Invest Dermatol. 2012;132(6):1591-1596.
Clinical, Cosmetic and Investigational Dermatology

\section{Publish your work in this journal}

Clinical, Cosmetic and Investigational Dermatology is an international, peer-reviewed, open access, online journal that focuses on the latest clinical and experimental research in all aspects of skin disease and cosmetic interventions. All areas of dermatology will be covered; contributions will be welcomed from all clinicians and

\section{Dovepress}

basic science researchers globally. This journal is indexed on CAS. The manuscript management system is completely online and includes a very quick and fair peer-review system, which is all easy to use. Visit http://www.dovepress.com/testimonials.php to read real quotes from published authors. 